\title{
What Works? Processes of Change in a Transdiagnostic Exposure Treatment for Patients With Chronic Pain and Emotional Problems
}

Martin Södermark, Steven J . Linton, Hugo Hesser, Ida Flink, Björn Gerdle and Katja Boersma

The self-archived postprint version of this journal article is available at Linköping University Institutional Repository (DiVA):

http:// urn.kb.se/ resolve?urn=urn:nbn:se:liu:diva-170672

N.B.: When citing this work, cite the original publication.

Södermark, M., Linton, S. J ., Hesser, H., Flink, I., Gerdle, B., Boersma, K., (2020), What Works? Processes of Change in a Transdiagnostic Exposure Treatment for Patients With Chronic Pain and Emotional Problems, The Clinical J ournal of Pain, 36(9), 648-657.

https:// doi.org/ 10.1097/ AJ P.0000000000000851

Original publication available at:

https:/ / doi.org/ 10.1097/AJ P.0000000000000851

Copyright: Lippincott, Williams \& Wilkins

http:// www.lww.com/ 


\section{What Works? Processes of Change in a Transdiagnostic Exposure Treatment for Patients with Chronic Pain and Emotional Problems.}

Martin Södermark, MSc, Pain and Rehabilitation Center and Department of Medical and Health Sciences, Linköping University, Sweden.

Steven J. Linton, Professor, PhD, Center for Health and Medical Psychology, Örebro University, Sweden.

Hugo Hesser, Professor, PhD, Center for Health and Medical Psychology, Örebro University, Sweden; Department of Behavioural Sciences and Learning, Division of Psychology, Linköping University, Sweden.

Ida Flink, PhD, Center for Health and Medical Psychology, Örebro University, Sweden.

Björn Gerdle, Professor, MD, PhD, Pain and Rehabilitation Center and Department of Medical and Health Sciences, Linköping University, Sweden.

Katja Boersma, Professor, PhD, Center for Health and Medical Psychology, Örebro University, Sweden.

Corresponding author: Martin Södermark, MSc, Pain and Rehabilitation Centre, Linköping University Hospital, 58185 Linköping, Sweden, e-mail: martin.sodermark@liu.se

The project received funding from AFA insurance (140356), Region Östergötland (LIO-724821) and the Swedish Foundation for Humanities and Social Sciences (P14-0799:1).

Disclosure: Data are also appearing in previous publication regarding primary outcomes ${ }^{1}$. An abstract and poster of preliminary results regarding primary outcomes was presented at the IASP world conference in Boston, USA 12-16 September 2018. 


\begin{abstract}
Objectives:

We recently developed a transdiagnostic exposure treatment ("the hybrid treatment") for chronic pain patients with concurrent emotional difficulties. This paper investigates the hypothesized treatment processes, specifically: a) if changes on pain-related dysregulation (catastrophizing, fear-avoidance and non-acceptance of pain) and general emotion dysregulation (difficulties to regulate a broad spectrum of emotional responses) mediate effects on outcomes; and b) if mediation is more pronounced for patients who score higher on these processes pre-treatment.
\end{abstract}

\title{
Methods:
}

Structural equation modeling for longitudinal data using the full intention-to-treat sample was used to test whether proposed variables mediated the effect of the hybrid treatment $(n=58)$ compared to a guided internet delivered pain management treatment based on cognitive-behavioral principles (iCBT; $n=57$ ) on pain interference and depressive symptoms at 9-months follow up. To make full use of the multiple process-measures collected in the trial, we modeled mediators as two continuous latent variables: pain-related dysregulation and general emotion dysregulation.

\section{Results:}

Reduced pain-related dysregulation mediated the effects of treatment on both outcomes, whereas reduced general emotion dysregulation mediated the effects on depressive symptoms only. In the hybrid treatment, the mediated effect was more pronounced for participants who scored higher on pain-related dysregulation pre-treatment relative to those who scored lower.

\section{Discussion:}

Our findings provide initial support for the transdiagnostic theoretical underpinnings of the hybrid treatment model. Using a hybrid treatment approach that centers on teaching patients emotion regulation skills before commencing broad exposure, successfully influenced both pain-related dysregulation and general emotion dysregulation, which in turn was associated with better treatment outcomes. It appears central to address these processes in pain patients with comorbid emotional problems, especially among patients scoring high on measures regarding catastrophizing, fear-avoidance and non-acceptance of pain.

Keywords: chronic pain; depression; cognitive-behavioral therapy; emotion regulation.

Trial registration: Preregistered at Clinicaltrails.gov (NCT02808286). 


\section{Introduction}

Cognitive Behavioral Therapy (CBT) has substantial empirical support based on a vast number of clinical trials and systematic reviews evaluating the effects on chronic pain ${ }^{1}$ as well as on emotional disturbances ${ }^{2}$. However, for the large number of patients suffering from concurrent pain and emotional problems, CBT treatments has shown to be less effective ${ }^{3-8}$. As comorbid pain and emotional problems entail a great deal of suffering, marginalization and societal costs, there is an urgent need for developing more effective treatments ${ }^{9-11}$.

One way to improve outcome for patients with comorbidities is to target transdiagnostic processes, underlying in this case both pain and emotional problems ${ }^{12}$. One potential transdiagnostic process is difficulties with emotion regulation, or emotion dysregulation. This has been conceptualized as involving; a) lack of awareness and understanding of emotions; b) nonacceptance of emotions; c) difficulties to control impulsive behaviours and behave in accordance with desired goals when experiencing emotions; and d) difficulties to use situationally-appropriate emotion regulation strategies flexibly in order to meet individual goals and situational demands ${ }^{13}$. Emotion dysregulation has been pinpointed as central in both psychiatric disorders ${ }^{14,15}$ as well as in chronic pain ${ }^{16}$. Several forms of recent CBT approaches explicitly this mechanism with the assumption that this might improve important outcomes such as well-being, health and disability 17,18. Building on these efforts, we developed a transdiagnostic exposure treatment ("the hybrid treatment") for patients with concurrent pain and emotional problems, integrating exposure methods based on the fear-avoidance model ${ }^{19}$ with an explicit emotion regulation approach informed by procedures in Dialectical Behavior Therapy ${ }^{20}$. Hence, the hybrid treatment rational

and protocol ${ }^{21}$ target transdiagnostic treatment processes, such as general skill deficits in coping with difficult emotions as well as pain-specific fears and catastrophizing thoughts. Hence, the protocol explicitly addresses both pain-related dysregulation and general emotion regulation difficulties.

After piloting the protocol in a controlled single case series ${ }^{22}$ we tested the hybrid treatment in a randomized controlled trial ${ }^{21}$, comparing it to a guided internet delivered pain management treatment based on CBT principles (iCBT). The hybrid treatment produced significantly better outcomes regarding depressive symptoms and pain interference at the 9-month follow-up. This initiates important follow up questions regarding how the treatment exerted its effect. Specifically, there is a need to understand the mediating variables, or processes by which change occurred ${ }^{23-25}$.

In accordance with the theoretical framework of the hybrid treatment, proposed mediators of change are: 1) reductions in pain-related dysregulation, altering cognitive, emotional and behavioral processes related to regulatory attempts to control and avoid pain - such as pain catastrophizing, non-acceptance and fear-avoidance beliefs; and 2) reductions in general emotion dysregulation by improving emotion regulation skills - such as awareness, understanding and acceptance of emotions, self-compassion, and ability to engage in goal-directed behavior. For the sake of brevity, these proposed mediators will be coined respectively 'pain-related dysregulation' and 'general emotion dysregulation'. Earlier treatment studies suggest that these variables do play important roles as mediators of pain outcomes.

Improvement on measures regarding pain-related dysregulation (such as: the Pain Catastrophizing Scale, PCS ${ }^{26}$; the Tampa Scale of Kinesiophobia, TSK ${ }^{27}$; and the Chronic Pain Acceptance Questionnaire, $\mathrm{CPAQ}^{28}$ ) have been shown to mediate effects on pain interference and disability in both CBT treatments and physical therapy for patients with chronic pain ${ }^{26-28}$, and reductions in emotion dysregulation (including several measures of difficulties in emotion regulation, rumination, and experiential avoidance) have been shown to correlate with effects on 
emotional problems such as depressive symptoms in multiple psychological treatments for psychiatric disorders ${ }^{14}$.

In the current study we used several measures to capture our proposed mediators. When exploring mediation in such a context, it is important to use a methodology that specifically addresses the overlap among variables, for both conceptual and methodological reasons ${ }^{29,30}$. One such approach, that we will apply in this study, is to reduce the number of overlapping observed variables to a smaller set of latent dimensions (i.e., latent variables) within the framework of structured equation modeling (SEM) ${ }^{31,32}$. This approach will also allow us to test important methodological assumptions of longitudinal mediation analysis (e.g. longitudinal measurement invariance) ${ }^{33}$, and overcome other problems associated with testing mediators with observed variables (e.g. measurement error) ${ }^{32}$.

The other important question we will address is whether the hybrid treatment exerts its effect through the same mechanism for all participants. Specifically, effects of treatments are often stronger for participants scoring worse on mediating variables before treatment (so called baseline moderated mediation) ${ }^{34}$. Therefore, we suggest that pre-treatment levels on pain-related dysregulation and general emotion dysregulation might moderate the mediated effect. By testing moderated mediation, we can determine for whom, and under what conditions, specific processes operate ${ }^{35}$. Information gained from such theory-driven analyses may further improve effectiveness of the hybrid treatment by identifying individuals who will benefit most from the treatment and by pointing out treatment techniques that change specific mediators for subgroups of individuals.

To summarize, in this study we focus on three hypotheses. First, we hypothesize that the hybrid treatment has a more pronounced effect than iCBT on the two latent process variables labeled pain-related dysregulation and general emotion dysregulation. Second, we hypothesize that effects on the latent variables at post-treatment mediate the effectiveness of the hybrid treatment on depressive symptoms and pain interference at follow-up. Our third hypothesis is that patients scoring worse on the mediator pre-treatment will have more pronounced indirect effects through that mediator. 


\section{Materials and Methods}

This study is based on a multicenter parallel group study in which 115 chronic pain patients with emotional problems were randomized to either the hybrid treatment $(n=58)$ or an active control condition receiving a guided internet-delivered pain management treatment based on CBT principles (iCBT; $n=57$ ). The current paper presents secondary analyses of the original RCT which main results are reported elsewhere ${ }^{21}$. The study was conducted between 2016 and 2018 at two sites in Sweden (Örebro and Linköping). The Ethics Review Board in Uppsala approved the study (2015/479) and the trial was preregistered at Clinicaltrails.gov (NCT02808286).

\section{Inclusion criteria}

We recruited chronic pain patients aged 18-70 via advertisements in local newspapers, social media and through clinical departments of pain rehabilitation. Inclusion criteria were: a) chronic musculoskeletal pain ( $>6$ months duration); b) functional problems in daily life due to pain ( $\geq 11$ points on items 21-24 of the Örebro Musculoskeletal Pain Questionnaire, ÖMPSQ ${ }^{36}$ ); c) emotional problems ( $\geq 8$ on either subscale of the Hospital Anxiety and Depression Scale, HADS ${ }^{37}$ ); $d$ ) access to computer or tablet; and e) sufficient mastery of the Swedish language. In addition, we excluded participants with: a) severe psychiatric disorders that may have required immediate or other treatment (alcohol abuse, psychotic disorders or at risk of suicide); b) ongoing psychological treatment elsewhere; and c) recently started, or changed, psychopharmacological treatment (cut off criterion: $<3$ months prior to planned treatment start).

We provide a more detailed description of the recruitment process in the original article presenting the results of the $\mathrm{RCT}^{21}$.

Table 1. Baseline description of participants' demographic and clinical characteristics.

\begin{tabular}{|c|c|c|}
\hline & Hybrid $(\mathrm{n}=58)$ & $\mathrm{iCBT}(\mathrm{n}=57)$ \\
\hline Gender, $N(\%$ women $)$ & $52(89.7 \%)$ & $44(77.2 \%)$ \\
\hline Age, mean (SD) & $45(12)$ & $44(12)$ \\
\hline $\begin{array}{c}\text { Screening measures, mean (SD) } \\
\text { - ÖMPSQ function }(0-40) \\
\text { - } \text { HADS anxiety }(0-21) \\
\text { - } \operatorname{HADS} \text { depression }(0-21)\end{array}$ & $\begin{array}{l}21(7.5) \\
12.2(4.0) \\
11.4(3.8)\end{array}$ & $\begin{array}{l}21.7(7.3) \\
11.2(4.1) \\
11.8(4.3)\end{array}$ \\
\hline $\begin{array}{l}\text { Occupational status, } N(\%) \\
\text { - } \text { Working } \\
\text { - Unemployed } \\
\text { - Student } \\
\text { - Pensioner } \\
\text { - Other }\end{array}$ & $\begin{array}{c}33(56.9 \%) \\
3(5.2 \%) \\
3(5.2 \%) \\
9(15.5 \%) \\
10(17.2 \%)\end{array}$ & $\begin{array}{c}34(59.6 \%) \\
4(7 \%) \\
4(7 \%) \\
6(10.5 \%) \\
9(15.8 \%)\end{array}$ \\
\hline $\begin{array}{cl}\text { Sick leave, } N(\% \text { during last year }) \\
\text { - } & 0-14 \text { days } \\
\text { - } & 15-180 \text { days } \\
\text { - } & 181-365 \text { days }\end{array}$ & $\begin{array}{l}25(43.1 \%) \\
8(13.8 \%) \\
25(43.1 \%)\end{array}$ & $\begin{array}{l}25(43.9 \%) \\
11(19.3 \%) \\
21(36.8 \%)\end{array}$ \\
\hline
\end{tabular}

Note. Hybrid; the hybrid treatment, iCBT; internet-delivered pain management treatment, ÖMPSQ; Örebro Musculoskeletal Pain Screening Questionnaire, item 21-24, HADS; Hospital Anxiety and Depression Scale. This data is also presented elsewhere ${ }^{1}$. 


\section{Sample}

Table 1 provides clinical and demographic characteristics of the included participants.

\section{Patient reported outcome measures}

Participants filled out assessment batteries electronically in their own environment, at pretreatment before randomization, at post-treatment (after median $=21, \mathrm{IQR}=17-26$ weeks) and at the 9-month follow up (after median $=60, \mathrm{IQR}=56-64$ weeks). All measures have been used, and most of them validated, in a Swedish context ${ }^{38-46}$.

Figure 1 shows the flow chart of the study procedure. The retention rate to post-treatment assessment was $81 \%$ for the hybrid treatment and $75 \%$ for $\mathrm{iCBT}$, and $79 \%$ and $84 \%$ respectively at follow up. In this report, we analyse assessment of mediators at pre and post-treatment, and outcome measures at pre-treatment and follow up.

\section{Outcomes}

To assess depressive symptoms, we used the Montgomery Åsberg Depression Rating Scale - Self report ${ }^{47}$ (MADRS-S; 9 items, range 0-60; test-retest reliability $r=.78^{48}$; Cronbach's $\alpha=.78$ ). To assess pain interference, we used the subscale from the Swedish version of the West HavenYale Multidimensional Pain Inventory ${ }^{49}$; MPI-interference (11 items, range 0-66; test-retest reliability $r=.85^{39}$; Cronbach's $\alpha=.87$ ). We replaced missing values regarding the item "interference with work" due to the participant not working, with the mean of the other items on the interference scale.

\section{Mediators}

As indicators for the latent variable pain-related dysregulation we used: 1) the Pain Catastrophizing Scale (PCS; 13 items, range 0-52; test-retest reliability $r=.75$ 50; Cronbach's $\alpha=.91$ ) assessing negative thinking related to actual or anticipated pain experiences ${ }^{50}$;2) the Tampa Scale for Kinesiophobia-11 (TSK-11; 11 items, range 11-44; test-retest reliability r=.81 ${ }^{51}$; Cronbach's $\alpha=.80$.) assessing fear of re-injury or worsening of pain due to movements and activities $^{52}$; and 3) the Chronic Pain Acceptance Questionnaire (CPAQ-8, 8 items, range 0-48, Cronbach's $\alpha=.78$ ) assessing the ability to engage in activities in the presence of pain, willingness to experience pain, and attempts to avoid or control pain ${ }^{53}$.

As indicators of the latent variable general emotion dysregulation we used: 1) the Difficulties in Emotion Regulation Scale (DERS; 36 items, range 36-180; test-retest reliability $\mathrm{r}=.88^{13}$; Cronbach's $\alpha=.93$ ) assessing a broad range of emotion regulation strategies including awareness of emotions, control of impulses, acceptance of emotions, access to functional regulation strategies and ability to pursue goals regardless of emotions ${ }^{13}$; 2) the Self-Compassion Scale-12 (SCS-12; 12 items, range 12-60, test-retest reliability $r=.89^{54}$, Cronbach's $\alpha=.87$ ) assessing emotion regulation strategies such as cognitive re-appraisal, self-compassion and acceptance ${ }^{55}$; and 3 ) the Behavioral Activation for Depression Scale (BADS; 25 items, range 0-150; test-retest reliability $\mathrm{r}=.74^{56}$, Cronbach's $\alpha=.85$ ) assessing difficulties with goal pursuit, avoidance behaviors, social withdrawal and rumination 56 .

The latent constructs aim to represent two relevant and theoretically distinct higher order factors; the first pain symptom specific coping and the second related to general emotion regulation abilities. The TSK, PCS and CPAQ, grouped under the first latent construct, all assess pain-specific cognitive-behavioral processes. While each instrument is associated to somewhat different theories, they have clear commonalities and overlap. Specifically, they all share the notion that pain triggers emotions and is associated with cognitions and behaviors that can be conceptualized as regulatory 
attempts to control and avoid pain ${ }^{51,53,57}$. The DERS, BADS and SCS, grouped under the second latent construct, all assess cognitive and behavioral attempts to regulate emotional responses $13,17,55,56$.

\section{Enrollment}

Assessed for eligibility $(n=399)$

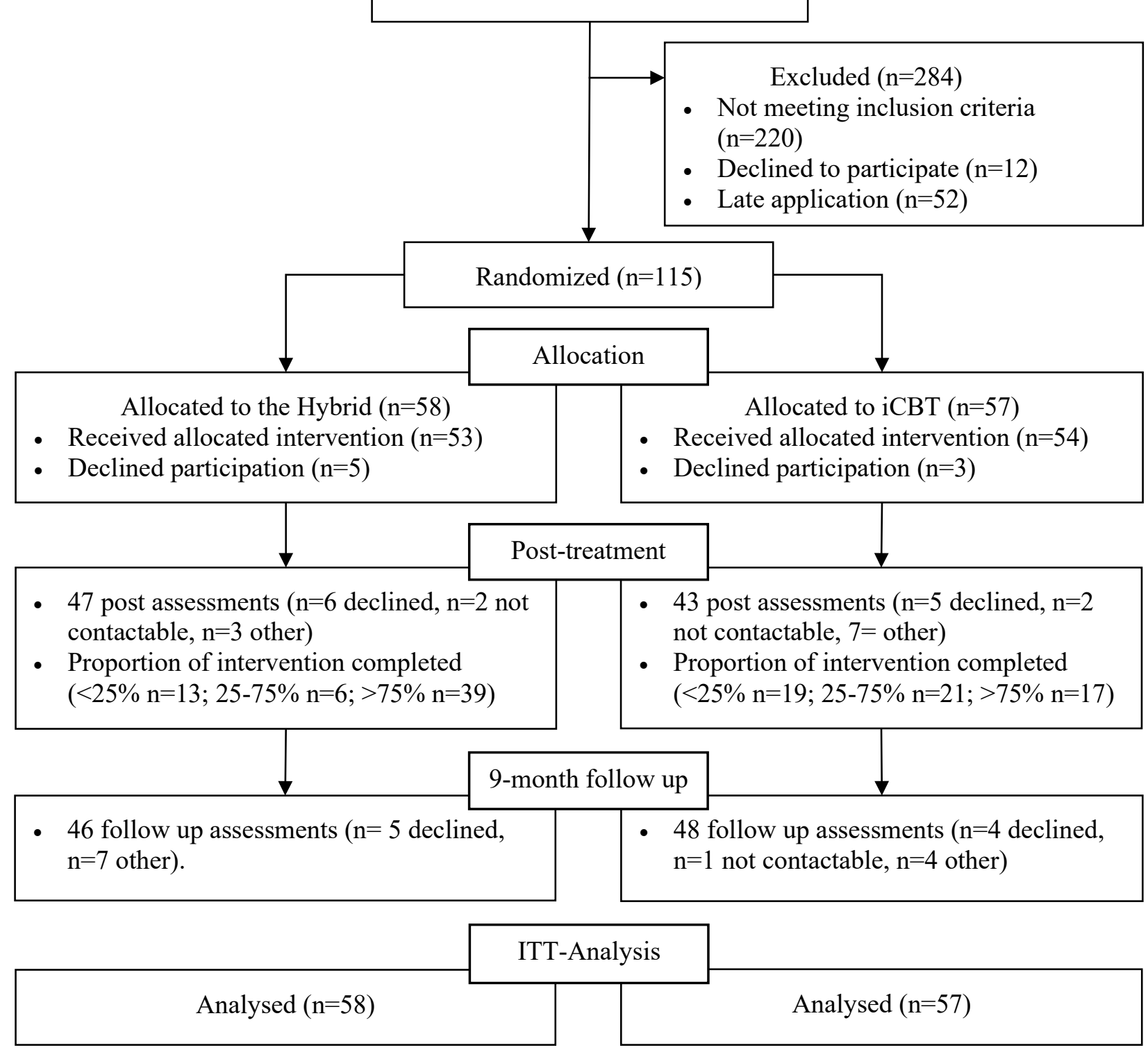

Figure 1. Flow chart of the study procedure. 


\section{Hybrid treatment}

The hybrid treatment is principle based and presented in five different treatment stages: 1) building a working relationship and developing relevant goals, 2) developing emotion regulation skills, 3) exposure for emotions and movements, 4) applying skills in tune with environment, and 5) maintaining and refining learned skills. A target of 10-15 weekly sessions was set, but since the stages allow for tailoring to the patients' needs, no specific formulations of session content, number of sessions per stage, or detailed step-by-step methods were provided. The treatment was conducted by licensed clinical psychologists $(n=5)$ and clinical psychologists in their post graduate year of supervised professional training for accreditation $(n=2)$.

\section{Internet-delivered pain management treatment (iCBT)}

The iCBT intervention included eight treatment modules consisting of educational texts, pictures, case examples, audio-files and two to three homework assignments per module. The content consisted of common cognitive behavioral interventions in pain-management such as graded exercise, pacing and activity planning, applied relaxation, coping with negative thoughts, mindfulness exercises, stress management, sleep hygiene and developing a maintenance plan. During treatment, participants were guided via the chat function of the internet platform by licensed clinical psychologists $(n=2)$, psychology students in their final stage of clinical training $(n=4)$ and clinical psychologists in their post academic year of supervised professional training for accreditation $(n=2)$.

\section{Statistical methods}

All primary data models were estimated within the framework of structural equation modeling (SEM) and fitted with full information maximum likelihood estimation with nonnormality robust standard errors (MLR or Bootstrap) using Mplus vs. $8.2^{58}$.

To make full use of multiple measures collected in the trial, we modeled mediators as two continuous latent variables. Indicators for the latent variable pain-related dysregulation were the total scores on TSK-11, PCS and CPAQ-8. Indicators for the latent variable general emotion dysregulation were total scores on DERS, SCS-12 and BADS. We reflected CPAQ-8, SCS-12 and BADS prior to analyses so that factor loadings were all positive, and higher scores on the factors were indicative of more dysfunction.

In addition to the advantages of using latent factors stated in the introduction (i.e., handling measurement error, empirical overlap among variables, test of measurement invariance) this approach also limited the number of tests required for testing mediation and thus prevented inflated type-1 error rates. Furthermore, by retaining information from all indicators and time points irrespectively of missing data for any individual, all randomized individuals were included in the primary models following the intention-to-treat principle using full information maximum likelihood, one of two recommended methods for handling missing data ${ }^{59}$.

We used the following procedures. First, to determine the adequacy of the proposed twofactor structure, we started by constructing confirmatory factor analysis (CFA) models, one at each time point (pre- and post-treatment). The cross-sectional CFA models were then combined into a longitudinal CFA model ${ }^{60}$. We evaluated the fit of the models using the chi-square test, where a good fitted model should not be rejected by the data as indicated by a non-statistically significant test. In addition, we used the Root Mean Square Error of Approximation (RMSEA), the Comparative Fit Index (CFI) and the Standardized Root Mean Square Residual (SRMR) with values $<.06^{61,62},>.95^{61,63}$, and $<.08^{61}$ as benchmark for a good fitted model. 
Second, we proceeded to test longitudinal measurement invariance for each latent variable (the two suggested mediators). Measurement invariance was constructed by constraining measurement intercepts and factor loadings for indicator variables for each latent factor to be equal across measurement points ${ }^{60,64}$. To test if the constraints significantly worsened model fit, we compared the global fit of the constrained model with a configural model in which these constraints were removed using a scaled chi-square difference test ${ }^{58,65}$. The more constrained model (null model) was deemed to fit worse than an alternative less restrictive model if the increase in chisquare statistics was statistically significant at the level of $p<.05$ (with degrees of freedom equal to the difference in free parameters between models). If this test was not statistically significant, we assumed longitudinal measurement invariance (i.e., scalar invariance) to be established. Following recommendations for longitudinal structural equation models ${ }^{60,64}$, residual errors were correlated for the same indicators across time to avoid model misspecifications.

Third, once factorial invariance was established, we could test for mediation by using the latent variables as mediators in a path model. The associations between $(\mathrm{X})$ treatment condition, (M) the latent variables as measured at post-treatment and (Y) the observed outcomes symptoms of depression and pain interference at 9-month follow up assessment, were modeled using regression among these continuous latent and observed variables. Specifically, the latent mediator variables post-treatment were regressed on the treatment condition ( $a$-path) as an observed binary coded variable $(1=$ hybrid treatment, $0=\mathrm{iCBT})$ and the observed outcome at 9 -month follow up was regressed on the latent mediator ( $b$-path). To control for initial scores on both the latent mediator and the observed outcome in the model, the mediator and outcome measured at posttreatment and follow up respectively, were regressed on the latent mediator variable and the outcome assessed at pre-treatment (similar to an ANCOVA model). By regressing the posttreatment value on the pre-treatment score, the net effect at post-treatment can be conceptualized as a change score between assessment points, and associations examined in mediator models can therefore be interpreted as predicting change in mediator and outcome (similar to how change is modeled in cross-lagged panel models ${ }^{66}$ ). This modeling approach also allowed us to control for bias due to regression toward the mean.

To formally evaluate mediation, we constructed bootstrapped confidence intervals around the product of the $a$ - and $b$-paths ( $a b$-product) from 5000 samples drawn with replacements ${ }^{34}$. If this asymmetric 95\% confidence interval did not contain zero, mediation was assumed to be established at the specified alpha level (5\%). To test for moderated mediation by pre-treatment levels on mediators, also known as mediated baseline by treatment moderation ${ }^{34,67}$, we created an interaction term between the latent variable measured at pre-treatment and the treatment condition variable. Moderated mediation was assumed to be established if the product between this regression due to the interaction (moderated $a$-path) and the regression due to association between mediator and outcome ( $b$-path) was statistically significant different from zero, as evaluated with the bootstrapped $95 \%$ asymmetric confidence interval method. To determine the size and significance of the effect, following recommendations for moderated mediation ${ }^{67}$, we probed a statistically significant moderated effect by calculating the conditional effect for a range of values of the moderator (values between $2 \mathrm{SD}$ above and below the mean). We then visualized the point estimate of the conditional effect along with a bootstrapped $95 \%$ confidence interval for each value of the moderator. 


\section{Results}

Table 2 shows the observed means and standard deviations of the sample on the indicator measures for the proposed latent variables, pain-related dysregulation and general emotional dysregulation, and on the outcome measures.

Table 2. Means and standard deviations (SD) at assessment points.

\begin{tabular}{|c|c|c|c|}
\hline Measure (range), treatment. & $\begin{array}{l}\text { Pre-treatment } \\
\text { M (SD) }\end{array}$ & $\begin{array}{l}\text { Post-treatment } \\
\text { M (SD) }\end{array}$ & $\begin{array}{l}\text { 9-month follow up } \\
\text { M (SD) }\end{array}$ \\
\hline \multicolumn{4}{|c|}{ Indicators for the latent variable pain-related dysregulation } \\
\hline $\begin{array}{l}\text { TSK-11 (11-44) } \\
\text { Hybrid } \\
\text { iCBT }\end{array}$ & $\begin{array}{l}24.34(6.47) \\
24.96(6.11)\end{array}$ & $\begin{array}{l}19.48(4.82) \\
24.0(6.08)\end{array}$ & $\begin{array}{l}\text { NA } \\
\text { NA }\end{array}$ \\
\hline $\begin{array}{l}\text { PCS }(0-52) \\
\text { Hybrid } \\
\text { iCBT }\end{array}$ & $\begin{array}{l}24.14(10.21) \\
26.86(10.54)\end{array}$ & $\begin{array}{c}16.98(9.97) \\
22.91(11.83)\end{array}$ & $\begin{array}{l}\text { NA } \\
\text { NA }\end{array}$ \\
\hline $\begin{array}{l}\text { CPAQ-8 }(0-48) \\
\text { Hybrid } \\
\text { iCBT }\end{array}$ & $\begin{array}{l}20.97(7.34) \\
20.25(7.98) \\
\end{array}$ & $\begin{array}{l}26.72(6.07) \\
22.12(7.59) \\
\end{array}$ & $\begin{array}{l}\text { NA } \\
\text { NA }\end{array}$ \\
\hline Indicators for the latent vari & neral emotion & ion & \\
\hline $\begin{array}{l}\text { DERS (36-180) } \\
\text { Hybrid } \\
\text { iCBT }\end{array}$ & $\begin{array}{l}94.64(25.85) \\
91.91(21.39)\end{array}$ & $\begin{array}{l}79.36(21.26) \\
83.23(24.98)\end{array}$ & $\begin{array}{l}\text { NA } \\
\text { NA }\end{array}$ \\
\hline $\begin{array}{l}\text { SCS-12 (12-60) } \\
\text { Hybrid } \\
\text { iCBT }\end{array}$ & $\begin{array}{l}32.05(9.89) \\
31.88(8.23)\end{array}$ & $\begin{array}{c}38.72(8.96) \\
35.83(10.66)\end{array}$ & $\begin{array}{l}\text { NA } \\
\text { NA }\end{array}$ \\
\hline $\begin{array}{l}\text { BADS }(0-150) \\
\text { Hybrid } \\
\text { iCBT }\end{array}$ & $\begin{array}{l}75.17(20.30) \\
79.04(18.67)\end{array}$ & $\begin{array}{l}93.77(23.36) \\
92.08(20.22)\end{array}$ & $\begin{array}{l}\text { NA } \\
\text { NA }\end{array}$ \\
\hline Outcomes* & & & \\
\hline $\begin{array}{l}\text { MADRS-S (0-60) } \\
\text { Hybrid } \\
\text { iCBT }\end{array}$ & $\begin{array}{l}23.72(7.62) \\
23.11(7.05)\end{array}$ & $\begin{array}{l}\text { NA } \\
\text { NA }\end{array}$ & $\begin{array}{l}15.33(9.63) \\
17.79(9.28)\end{array}$ \\
\hline $\begin{array}{l}\text { MPI- Interference (0-66) } \\
\text { Hybrid } \\
\text { iCBT }\end{array}$ & $\begin{array}{l}49.63(10.46) \\
48.62(12.09)\end{array}$ & $\begin{array}{l}\text { NA } \\
\text { NA }\end{array}$ & $\begin{array}{l}36.39(16.30) \\
41.32(16.47)\end{array}$ \\
\hline
\end{tabular}

Note. Hybrid; the hybrid treatment, iCBT; internet-delivered pain management treatment. TSK-11; Tampa Scale for Kinesiophobia-11, PCS; Pain-catastrophizing scale, CPAQ-8; Chronic Pain Acceptance Questionnaire-8. DERS; Difficulties in Emotion Regulation Scale, SCS-12; Self-Compassion Scale-12, BADS; Behavioral Activation for Depression Scale. MADRS-S; Montgomery Åsberg Depression Rating Scale, MPI; West Haven-Yale Multidimensional Pain Inventory. Pre N for Hybrid=58, for iCBT N=57; post $\mathrm{N}$ for Hybrid=47, for $\mathrm{iCBT} \mathrm{N}=43$; follow-up $\mathrm{N}$ for Hybrid=46, for $\mathrm{iCBT}=48$. NA = not applicable. *Data on outcomes also presented elsewhere ${ }^{1}$. 


\section{Factor structure invariance}

In preparation of mediation analyses, we first determined the adequacy of the proposed twofactor structure and tested longitudinal measurement invariance. Fit indices obtained from both the cross-sectional two-factor CFA models and the longitudinal CFA model are presented in Table 3. Standardized factor loadings for indicators and correlation between latent factors are provided in the supplementary figure. Fit indices indicated that the statistical models fitted the data well, and factor loadings for the indicators were all high (>.62), supporting the use of latent factors. The estimated correlation between the latent factors, pain-related dysregulation and general emotion dysregulation, was moderate pre-treatment $(r=.30)$ and large post-treatment $(r=.67)$.

Separate tests of longitudinal measurement invariance revealed that constraints on factor loadings and intercepts across measurement points did not significantly degrade model fit for the latent variables (pain-related dysregulation: $\Delta \chi^{2}(4)=0.829, p=.934$; general emotion dysregulation: $\Delta \chi^{2}(4)=9.073, p=.06$ ). This suggested that longitudinal measurement invariance was established, and we could proceed to test for mediation by using the latent variables as mediators in a path model. Since the high correlation between latent factors at post-treatment could cause multicollinearity problems, we tested for mediation for each latent variable separately.

Table 3. Fit indices for estimated confirmatory factor analysis models for evaluating latent variables crosssectionally and longitudinally.

\begin{tabular}{lcccccc} 
Model & Chi-2 & df & CFI & SRMR & RMSEA & {$[90 \%$ CI $]$} \\
\hline Two factor model pre-treatment & $6.03^{\text {ns }}$ & 8 & 1.00 & 0.04 & 0.000 & {$[0.000,0.09]$} \\
Two factor model post-treatment & $7.77^{\text {ns }}$ & 8 & 1.00 & 0.034 & 0.000 & {$[0.000,0.12]$} \\
Longitudinal two factor model & $58.93^{\text {ns }}$ & 50 & 0.99 & 0.079 & 0.039 & {$[0.000,0.075]$} \\
\hline
\end{tabular}

Note. The longitudinal two factor model constrained factor loadings and intercepts to be equal across measurement points (scalar invariance). CFI = Comparative Fit Index; SRMR $=$ Standardized Root Mean Residual; RMSEA = Root Mean-Square Error of Approximation; CI=Confidence interval.

ns $=$ non-significant.

\section{Mediation}

Figure 2 displays the results from the estimated mediation models. There was a statistically significant effect of treatment condition on post-treatment pain-related dysregulation and general emotion dysregulation (covarying pre-treatment scores on the mediator and outcome). This indicates that the hybrid treatment, on average, reduced pain-related dysregulation and general emotion dysregulation relative to iCBT ( $a$-path).

There were also statistically significant effects of pain-related dysregulation ( $b$-path) on depressive symptoms and pain interference at 9-month follow up (primary endpoint), and for general emotion dysregulation (b-path) on depressive symptoms but not on pain interference at 9month follow up (primary endpoint). This indicates that larger treatment effects on pain-related dysregulation post-treatment were associated with reduced scores on pain interference and depressive symptoms at follow up, and larger treatment effects on general emotion dysregulation were associated with reduced scores on depressive symptoms.

Subsequently, pain-related dysregulation was a statistically significant mediator of the effect of the hybrid treatment on both depressive symptoms and pain interference, whereas general emotion dysregulation only mediated the hybrid treatment effect on depressive symptoms. 

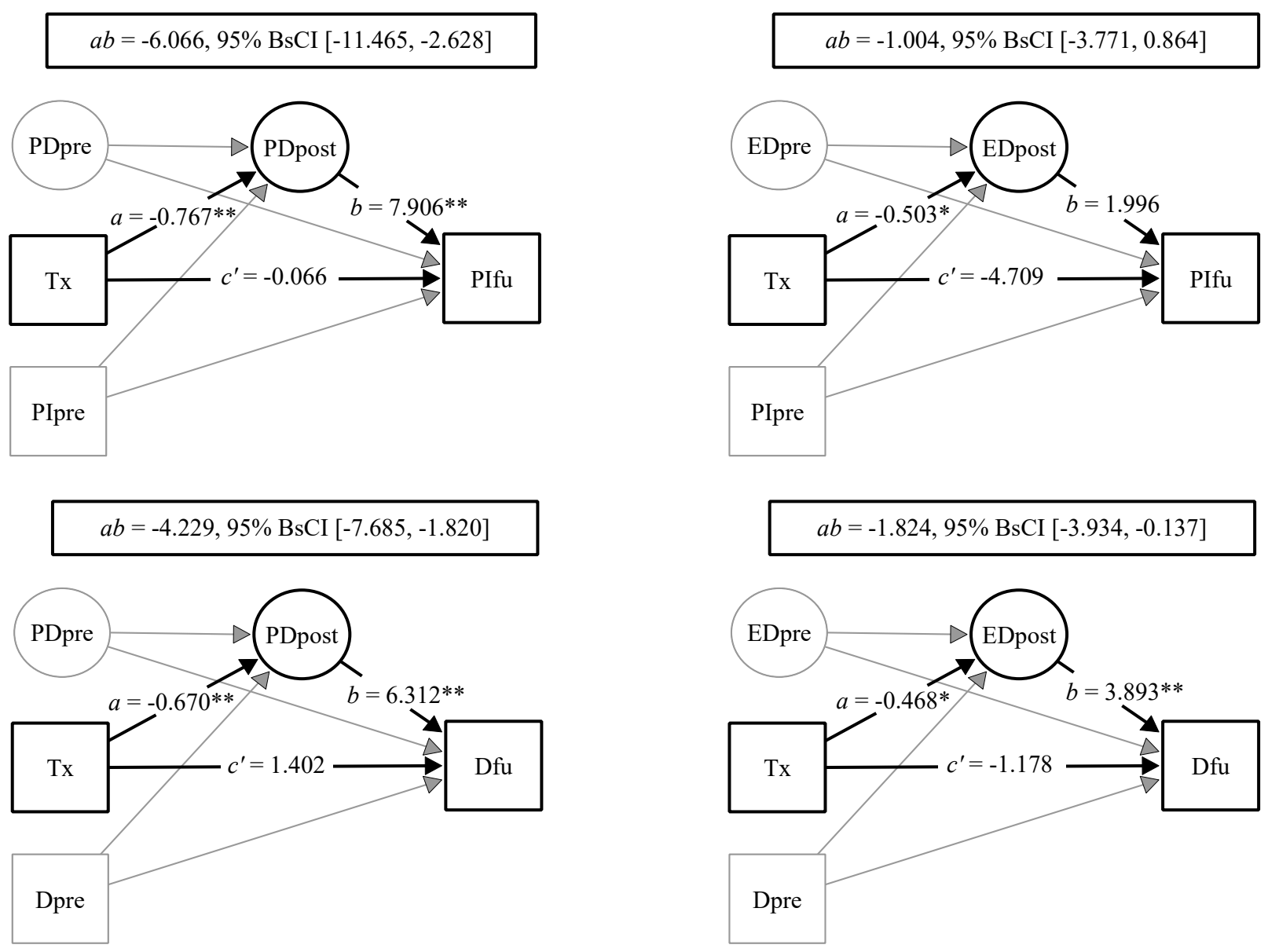

Figure 2. Estimated mediation models with robust maximum likelihood parameter estimates, beta coefficients for the associations among treatment condition, latent mediators, and outcomes, along with tests of indirect effects (ab-product with 95\% asymmetric bootstrap confidence intervals). Observed indicators for latent variables (circles) and residual covariance are not shown. PDpre $=$ pain-related dysregulation pre-treatment; PDpost $=$ pain-related dysregulation post-treatment; EDpre $=$ general emotion dysregulation pre-treatment; EDpost $=$ general emotion dysregulation post-treatment. $\mathrm{Tx}=$ binary treatment variable $(1=$ Hybrid, $0=\mathrm{iCBT})$; PIpre = pain interference pre-treatment; PIfu= pain interference at 9-month follow up; Dpre = depressive symptoms pre-treatment; Dfu = depressive symptoms at 9-month follow up. $*=\mathrm{p}<.05, * *=\mathrm{p}<.01$. 


\section{Baseline moderated mediation}

As a final step we tested whether the effect of the hybrid treatment was moderated by pretreatment levels on pain-related dysregulation and general emotion dysregulation (so called baseline moderated mediation). The model with depressive symptoms as outcome and pain-related dysregulation as mediator, revealed a statistically significant interaction effect between treatment condition and pain-related dysregulation at pre-treatment on the mediator, pain-related dysregulation, measured at post-treatment (beta $=-0.479, \mathrm{SE}=0.191, \mathrm{z}=2.513, p=.012$ ). The moderated mediation effect, as evaluated by the product between this interaction term and the association between mediator and outcome, was also statistically significant (beta $=-3.052,95 \%$ CI [-6.232, -0.621]).

Figure 3 visualizes the conditional treatment effect on the mediator, as a function of scores between two standard deviations above and below the grand mean of pain-related dysregulation measured at pre-treatment. As can be seen, the difference between the hybrid treatment and iCBT increased as a function of higher scores on pain-related dysregulation at pre-treatment. This means that higher scores on pain-related dysregulation at pre-treatment were associated with a stronger positive effect of the hybrid treatment on the mediator pain-related dysregulation at post-treatment. For example, for participants scoring 1 standard deviation above the mean at pre-treatment (indicating more pain-related dysregulation) the effect was statistically significant and of substantial magnitude, as indicated by a $d=1.18$-point estimate of the standardized mean difference between treatment and control. However, for participants scoring approximately 0.7 standard deviation below the mean at pre-treatment (indicating less pain-related dysregulation), the difference between the hybrid treatment and iCBT was not statistically significant.

The same pattern of results was observed in the model examining moderated mediation with pain-related dysregulation as a mediator and pain interference as the outcome: the product term for testing moderated mediation was statistically significant (beta $=-3.285,95 \%$ CI [-7.081, 0.298]). This means that higher scores on pain-related dysregulation at pre-treatment were associated with a stronger positive effect of the hybrid treatment on the mediator pain-related dysregulation at post-treatment, and the effect on pain-related dysregulation at post-treatment were subsequently correlated with pain interference at 9-month follow-up.

The models with general emotion dysregulation as the mediator revealed no statistically significant interaction effects between treatment condition and general emotion dysregulation at pre-treatment on general emotion dysregulation assessed at post-treatment ( $p$ >.56). Accordingly, the product for evaluating moderated mediation was not statistically significant in either of the models examining general emotion dysregulation as the mediator (pain interference model: beta = $-0.295,95 \%$ CI [-2.389, 1.828]; depressive symptoms model: beta $=-0.62195 \%$ CI [-2.982, 2.241]. Thus, in contrast to pain-related dysregulation, the mediated effect of general emotional dysregulation was not dependent on pre-treatment levels on general emotional dysregulation. 


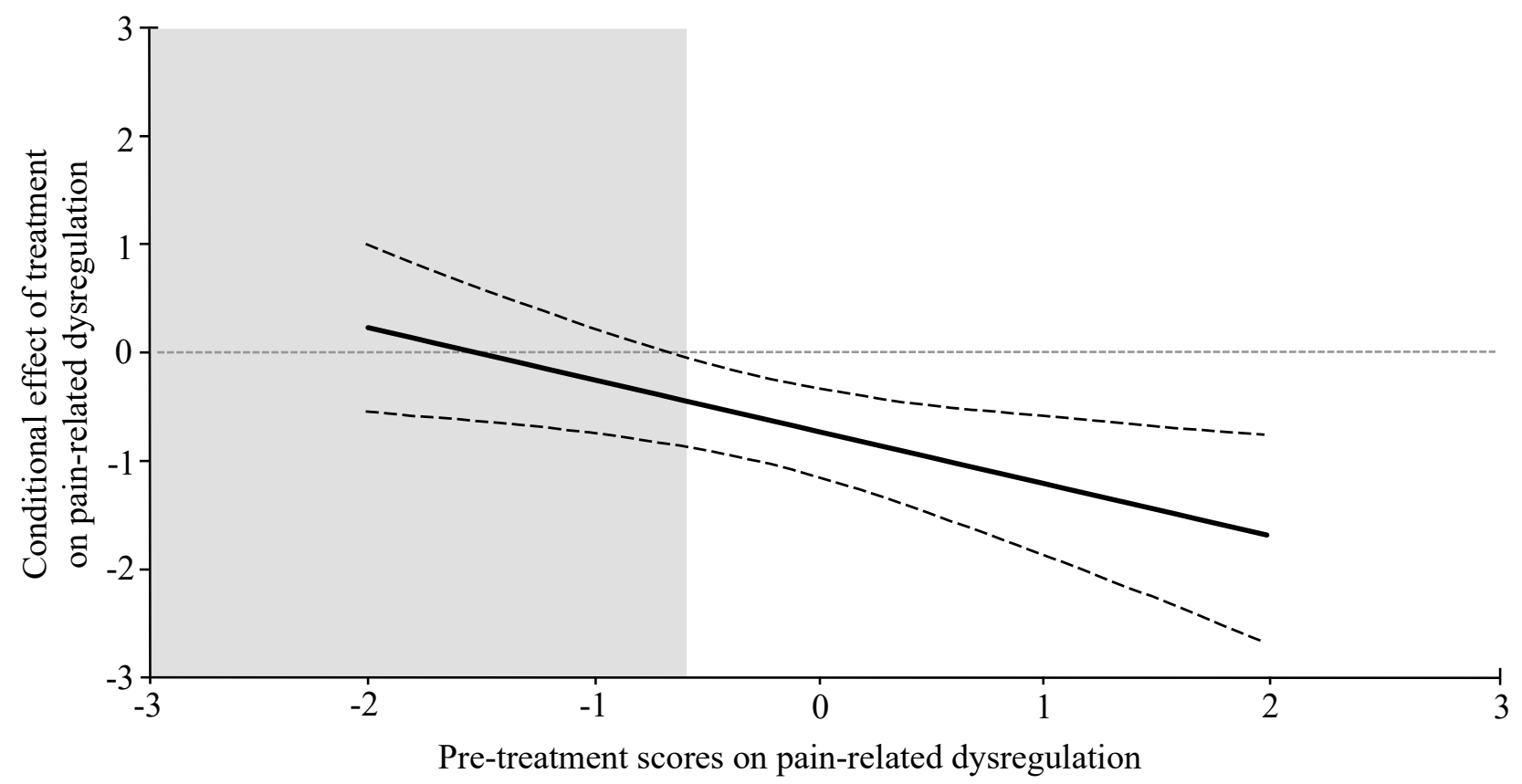

Figure 3. The conditional standardized treatment effect (d) on the latent mediator pain-related dysregulation at post-treatment as a function of pre-treatment scores on the same latent variable. The solid line represents the point estimate and the dashed lines represent lower and upper limit of bootstrapped $95 \%$ confidence intervals. The shaded grey area represents the region of non-significance. 


\section{Discussion}

In this study we aimed to test the theoretical underpinnings of the hybrid treatment, using data from a previously published randomized controlled trial comparing it to iCBT ${ }^{21}$. Results from our analysis were largely consistent with the theoretical model, supporting several of our hypotheses. First, and foremost, the hybrid treatment was more effective than iCBT in influencing both proposed mediators: pain-related dysregulation and general emotion dysregulation. Moreover, we found that both mediated effects on outcome. Specifically, improved pain-related dysregulation and general emotion dysregulation mediated effects on depressive symptoms, and improved painrelated dysregulation mediated effects on pain interference. Hence, we conclude that the previously reported effectiveness of this transdiagnostic exposure treatment for pain patients with concurrent emotional problems, at least partially, can be attributed to its effect on cognitive, emotional and behavioral processes related to regulatory attempts to control and avoid pain, and general emotion dysregulation.

These findings are in line with studies showing that improved catastrophizing and painacceptance mediate effects on pain interference in similar cognitive-behavioral treatments focusing on exposure, values and pain-coping ${ }^{26-28}$. In addition, these results add to the increasing empirical support for the theoretical assumption that pain interference, catastrophizing, inactivity and lack of reinforcement are important factors in the development and maintenance of depression in the context of chronic pain ${ }^{8,68,69}$.

The other important hypothesis addressed in this study was that mediation effects would be moderated by patients' levels of pre-treatment pain-related dysregulation and general emotion dysregulation. We found partial support for this idea. Individuals scoring higher on pain-related dysregulation showed a more pronounced change on the mediator relative to those scoring lower, thereby moderating the indirect effect of pain-related dysregulation on both depressive symptoms and pain interference. Not only does this finding provide further support for pain-related dysregulation as an important mediator of the hybrid treatment, but it also gives prescriptive information that can guide further developments of tailored treatments and a direction for efforts to answer the clinical question: what works for whom? Specifically, it indicates that the hybrid treatment may be a superior treatment for pain patients with concurrent emotional problems who also have high levels of pain-related dysregulation and avoidance behaviors, a group of patients that was found to have suboptimal treatment results with standard exposure treatment ${ }^{4}$.

Contrary to our hypothesis however, we found that mediation was not dependent on patients' pre-treatment level of general emotion dysregulation. This may indicate that, while change in depressive symptoms is mediated by improved emotion regulation, this process might be important regardless of initial emotion regulation problem levels. This result is in line with a recent review by Sloan et al. ${ }^{14}$, noting that emotion dysregulation is an important process in several cognitive behavioral treatments for patients with psychiatric disorders. However, the lack of moderated mediation could be a result of sample selection. As only patients with emotional problems were included, and as emotion dysregulation and depressive symptoms are closely connected, this may have restricted the variance in emotion dysregulation problems and thus influenced results.

In our analyses, we chose to condense a total of six potential mediators under the umbrella of two latent variables that we labelled 'pain-related dysregulation' and 'general emotion dysregulation'. With this we aimed to capture, on the one hand, pain-specific cognitive, emotional and behavioral processes and, on the other hand, general emotion regulation skills. While, arguably, nuances in psychological processes and specificity of constructs may be compromised, we did so for specific reasons. First, the three constructs and measures underlying each respective latent 
variable are conceptually interrelated and can as such represent two relevant and theoretically distinct higher order factors: one symptom pain specific coping and the one related to general emotion regulation abilities. By condensing constructs we wanted to acknowledge the growing concern that seemingly different psychological constructs in fact capture the same behavioral patterns ${ }^{70,71}$. In addition, clustering process measures in two latent variables was determined to be statistically advantageous and we could confirm that the models fitted the data well, both crosssectionally and longitudinally. Still, these choices do not imply that no other valid options are available, and several other models may have fitted the data well. For example, a high correlation was observed between the two latent factors at post-assessment that suggests the existence of a higher-order factor (e.g., general dysfunctional coping strategies) that could potentially account for an additional amount of the shared variance among the latent factors. While we deemed that the two latent constructs represented relevant and distinct theoretical entities, this finding reinforces the issue of the considerable empirical overlap in psychological constructs and that modeling each variable as a separate mediator would be more problematic.

The results of this study should be interpreted considering its limitations. Even though randomization strengthens claims of effects of treatment on mediators and outcomes, further research is warranted to confirm causality regarding indirect effects in the association between mediators and outcomes (b-path). On a related note, although the time lag between mediators (assessed at post-treatment) and outcomes (assessed at follow-up) strengthen the findings from our mediation models, we cannot be certain that effects on mediators precede effects on outcomes as most of the improvements in outcomes had already occurred at post-assessment ${ }^{34}$. Future studies may include repeated measures to establish the temporal relation between mediators and outcomes so that changes in mediators more reliably precede, and can be concluded to contribute to, subsequent changes in outcome. We also acknowledge that the sample size is in the lower range for the applied statistical models ${ }^{72}$. This may have influenced model fit statistics and stability of parameter estimates. However, we believe the benefits of these models surpass their disadvantage. As detailed in the introduction and method section, this approach allowed us to overcome problems associated with measurement error, factorial non-invariance, type-1 error rates, and missing data. Indeed, the use of latent factors in SEM may be especially important in mediation analysis due to these reasons ${ }^{31,32}$. It is also important to remember that all variables rely on self-report instruments which, although theoretically distinct, are empirically related with a natural overlap. For instance, catastrophizing might be regarded as a potential mediator, but it may also be a symptom of depression, e.g. one aspect of the outcome. Nevertheless, our study is based on a theoretical distinction between process variables and outcomes, and the findings should be interpreted keeping that in mind.

In conclusion, this study adds to the theoretical understanding of, and advancements in, developing treatments for chronic pain patients with concurrent emotional problems. One important finding is that using a hybrid treatment approach that centers on improving patients' emotion regulation skills, combined with broad exposure in-vivo, successfully influenced painrelated dysregulation and general emotion dysregulation and resulted in better treatment outcomes. Thus, addressing these processes appears central for pain patients with chronic pain and comorbid emotional problems. Another important finding points at a need to consider patients baseline levels of pain-related dysregulation, such as catastrophizing, fear-avoidance and non-acceptance of pain, in order to match treatment and optimize treatment results. Although further studies are warranted on the issue of moderation of outcomes, the results imply that the hybrid might have most added value for this subgroup of individuals. 
Our study has tapped into the complex, but essential question of what works for whom, how, and in what context. This is a first step in discovering how we best target transdiagnostic processes to enhance treatment outcomes. 


\section{Acknowledgments}

The authors would like to acknowledge all participants in this study, as well as study coordinators Maria Lind, Sara Nygren and Sara Edlund. We would also like to acknowledge Dr. Monica Buhrman for generously sharing the content of the internet treatment used in this study. Funding was provided by AFA insurance (140356), Region Östergötland (LIO-724821) and the Swedish Foundation for Humanities and Social Sciences (P14-0799:1). The content of this study is the sole responsibility of the authors; financial sponsors had no role in study design, data collection, data analysis, data interpretation, writing of the report, or the decision to submit for publication. The authors report no conflicts of interest. 


\section{References}

1. Eccleston C, Morley SJ, Williams AC. Psychological approaches to chronic pain management: evidence and challenges. Br J Anaesth. 2013;111(1):59-63.

2. Hofmann SG, Asnaani A, Vonk IJJ, Sawyer AT, Fang A. The efficacy of cognitive behavioral therapy: A review of meta-analyses. Cognit Ther Res. 2012;36(5):427-440.

3. Morley S, Williams A, Hussain S. Estimating the clinical effectiveness of cognitive behavioural therapy in the clinic: Evaluation of a CBT informed pain management programme. Pain. 2008;137(3):670-680.

4. Flink IK, Boersma K, Linton SJ. Catastrophizing moderates the effect of exposure in vivo for back pain patients with pain-related fear. Eur J Pain. 2010;14(8):887-892.

5. Bergbom S, Boersma K, Overmeer T, Linton SJ. Relationship Among Pain Catastrophizing, Depressed Mood, and Outcomes Across Physical Therapy Treatments. Phys Ther. 2011;91(5):754-764.

6. Flink IK, Boersma K, Linton SJ. Changes in catastrophizing and depressed mood during and after early cognitive behaviorally oriented interventions for pain. Cogn Behav Ther. 2014;43(4):332341.

7. Asmundson GJ, Katz J. Understanding the co-occurrence of anxiety disorders and chronic pain: state-of-the-art. Depress Anxiety. 2009;26(10):888-901.

8. Bair MJ, Robinson RL, Katon W, Kroenke K. Depression and pain comorbidity: a literature review. Arch Intern Med. 2003;163(20):2433-2445.

9. Boersma K, Linton SJ. Screening to identify patients at risk - Profiles of psychological risk factors for early intervention. Clin J Pain. 2005;21(1):38-43.

10. Sullivan MJL, Adams H, Thibault P, Corbiere M, Stanish WD. Initial depression severity and the trajectory of recovery following cognitive-behavioral intervention for work disability. J Occup Rehabil. 2006;16(1):63-74.

11. Phillips C, Main C, Buck R, Aylward M, Wynne-Jones G, Farr A. Prioritising pain in policy making: The need for a whole systems perspective. Health Policy. 2008;88(2):166-175.

12. Linton SJ. Applying dialectical behavior therapy to chronic pain: a case study. Scandinavian Journal of Pain. 2010;1(1):50-54.

13. Gratz KL, Roemer L. Multidimensional Assessment of Emotion Regulation and Dysregulation: Development, Factor Structure, and Initial Validation of the Difficulties in Emotion Regulation Scale. Journal of Psychopathology and Behavioral Assessment. 2004;26(1):41-54.

14. Sloan E, Hall K, Moulding R, Bryce S, Mildred H, Staiger PK. Emotion regulation as a transdiagnostic treatment construct across anxiety, depression, substance, eating and borderline personality disorders: A systematic review. Clin Psychol Rev. 2017;57(Supplement C):141-163.

15. Aldao A, Gee DG, De Los Reyes A, Seager I. Emotion regulation as a transdiagnostic factor in the development of internalizing and externalizing psychopathology: Current and future directions. Dev Psychopathol. 2016;28(4):927-946.

16. Linton SJ. A Transdiagnostic Approach to Pain and Emotion. J Appl Biobehav Res. 2013;18(2):82-103.

17. Gross JJ. Handbook of emotion regulation. Second Edition. ed. New York: The Guilford Press; 2014.

18. Lumley MA, Schubiner H. Emotional Awareness and Expression Therapy for Chronic Pain: Rationale, Principles and Techniques, Evidence, and Critical Review. Curr Rheumatol Rep. 2019;21(7):30.

19. Vlaeyen JW, Linton SJ. Fear-avoidance model of chronic musculoskeletal pain: 12 years on. Pain. 2012;153(6):1144-1147.

20. Koerner K. Doing diablectical behavior therapy: a practical guide. New York: The Guilford Press; 2012. 
21. Boersma K, Södermark M, Hesser H, Flink IK, Gerdle B, Linton SJ. Efficacy of a transdiagnostic emotion-focused exposure treatment for chronic pain patients with comorbid anxiety and depression. Pain. 2019;160(8):1708-1718.

22. Linton SJ, Fruzzetti A. A hybrid emotion-focused exposure treatment for chronic pain: A feasibility study. Scandinavian Journal of Pain. 2014;5(3):151-158.

23. Agler R, De Boeck P. On the Interpretation and Use of Mediation: Multiple Perspectives on Mediation Analysis. Front Psychol. 2017;8:1984.

24. Hayes AF, Rockwood NJ. Regression-based statistical mediation and moderation analysis in clinical research: Observations, recommendations, and implementation. Behav Res Ther. 2017;98:39-57.

25. Morley S, Keefe FJ. Getting a handle on process and change in CBT for chronic pain. Pain. 2007;127(3):197-198.

26. Smeets RJ, Vlaeyen JW, Kester AD, Knottnerus JA. Reduction of pain catastrophizing mediates the outcome of both physical and cognitive-behavioral treatment in chronic low back pain. $J$ Pain. 2006;7(4):261-271.

27. Schemer L, Schroeder A, Ørnbøl E, Glombiewski JA. Exposure and cognitive-behavioural therapy for chronic back pain: An RCT on treatment processes. European Journal of Pain (United Kingdom). 2019;23(3):526-538.

28. Cederberg JT, Cernvall M, Dahl J, von Essen L, Ljungman G. Acceptance as a Mediator for Change in Acceptance and Commitment Therapy for Persons with Chronic Pain? Int J Behav Med. 2016;23(1):21-29.

29. Cheung GW, Lau RS. Testing Mediation and Suppression Effects of Latent Variables: Bootstrapping With Structural Equation Models. Organizational Research Methods. 2007;11(2):296-325.

30. Holmbeck GN. Toward terminological, conceptual, and statistical clarity in the study of mediators and moderators: Examples from the child-clinical and pediatric psychology literatures. J Consult Clin Psychol. 1997;65(4):599-610.

31. Cheung GW, Lau RS. Accuracy of Parameter Estimates and Confidence Intervals in Moderated Mediation Models. Organizational Research Methods. 2015;20(4):746-769.

32. Muthén B, Asparouhov T. Causal Effects in Mediation Modeling: An Introduction With Applications to Latent Variables. Structural Equation Modeling: A Multidisciplinary Journal. 2014;22(1):12-23.

33. McArdle JJ. Latent Variable Modeling of Differences and Changes with Longitudinal Data. Annu Rev Psychol. 2009;60:577-605.

34. MacKinnon DP, Fairchild AJ, Fritz MS. Mediation analysis. Annu Rev Psychol. 2007;58:593614.

35. Tein JY, Sandler IN, MacKinnon DP, Wolchik SA. How did it work? Who did it work for? Mediation in the context of a moderated prevention effect for children of divorce. J Consult Clin Psychol. 2004;72(4):617-624.

36. Linton SJ, Boersma K. Early identification of patients at risk of developing a persistent back problem: The predictive validity of the Orebro Musculoskeletal Pain Questionnaire. Clin J Pain. 2003;19(2):80-86.

37. Zigmond AS, Snaith RP. The hospital anxiety and depression scale. Acta Psychiatr Scand. 1983;67(6):361-370.

38. Buer N, Linton SJ. Fear-avoidance beliefs and catastrophizing: occurrence and risk factor in back pain and ADL in the general population. Pain. 2002;99(3):485-491.

39. Bergström G, Jensen IB, Bodin L, Linton SJ, Nygren AL, Carlsson SG. Reliability and factor structure of the multidimensional pain inventory - Swedish language version (MPI-S). Pain. 1998;75(1):101-110. 
40. Johansson R, Carlbring P, Heedman A, Paxling B, Andersson G. Depression, anxiety and their comorbidity in the Swedish general population: point prevalence and the effect on health-related quality of life. PeerJ. 2013;1.

41. Svanborg P, Asberg M. A comparison between the Beck Depression Inventory (BDI) and the self-rating version of the Montgomery Asberg Depression Rating Scale (MADRS). J Affect Disord. 2001;64(2-3):203-216.

42. Roelofs J, Sluiter JK, Frings-Dresen MH, et al. Fear of movement and (re)injury in chronic musculoskeletal pain: Evidence for an invariant two-factor model of the Tampa Scale for Kinesiophobia across pain diagnoses and Dutch, Swedish, and Canadian samples. Pain. 2007;131(1-2):181-190.

43. Bratt A, Fagerstrom C. Self-compassion in old age: confirmatory factor analysis of the 6-factor model and the internal consistency of the Self-compassion scale-short form. Aging Ment Health. 2019:1-7.

44. Rovner GS, Arestedt K, Gerdle B, Borsbo B, McCracken LM. Psychometric properties of the 8item Chronic Pain Acceptance Questionnaire (CPAQ-8) in a Swedish chronic pain cohort. $J$ Rehabil Med. 2014;46(1):73-80.

45. Björstad S, Johansson M. Validation of the Behavioral Activation for Depression Scale (BADS) in a student sample and a sample with elevated depressive symptoms (Master's thesis). 2008.

46. Norlund F, Wallin E, Olsson EMG, et al. Internet-Based Cognitive Behavioral Therapy for Symptoms of Depression and Anxiety Among Patients With a Recent Myocardial Infarction: The U-CARE Heart Randomized Controlled Trial. J Med Internet Res. 2018;20(3):e88.

47. Zimmerman M, Chelminski I, Posternak M. A review of studies of the Montgomery-Asberg Depression Rating Scale in controls: implications for the definition of remission in treatment studies of depression. Int Clin Psychopharmacol. 2004;19(1):1-7.

48. Fantino B, Moore N. The self-reported Montgomery-Åsberg depression rating scale is a useful evaluative tool in major depressive disorder. BMC Psychiatry. 2009;9:26-26.

49. Kerns RD, Turk DC, Rudy TE. The West Haven-Yale Multidimensional Pain Inventory (WHYMPI). Pain. 1985;23(4):345-356.

50. Sullivan MJL, Bishop SR, Pivik J. The Pain Catastrophizing Scale: Development and validation. Psychol Assess. 1995;7(4):524-532.

51. Hapidou EG, O'Brien MA, Pierrynowski MR, de Las Heras E, Patel M, Patla T. Fear and Avoidance of Movement in People with Chronic Pain: Psychometric Properties of the 11-Item Tampa Scale for Kinesiophobia (TSK-11). Physiother Can. 2012;64(3):235-241.

52. Woby SR, Roach NK, Urmston M, Watson PJ. Psychometric properties of the TSK-11: a shortened version of the Tampa Scale for Kinesiophobia. Pain. 2005;117(1-2):137-144.

53. Fish RA, McGuire B, Hogan M, Morrison TG, Stewart I. Validation of the chronic pain acceptance questionnaire (CPAQ) in an Internet sample and development and preliminary validation of the CPAQ-8. Pain. 2010;149(3):435-443.

54. Garcia-Campayo J, Navarro-Gil M, Andrés E, Montero-Marin J, López-Artal L, Demarzo MMP. Validation of the Spanish versions of the long (26 items) and short (12 items) forms of the SelfCompassion Scale (SCS). Health and quality of life outcomes. 2014;12:4-4.

55. Raes F, Pommier E, Neff KD, Van Gucht D. Construction and factorial validation of a short form of the Self-Compassion Scale. Clin Psychol Psychother. 2011;18(3):250-255.

56. Kanter JW, Mulick PS, Busch AM, Berlin KS, Martell CR. The Behavioral Activation for Depression Scale (BADS): Psychometric properties and factor structure. Journal of Psychopathology and Behavioral Assessment. 2007;29(3):191-202.

57. Flink IK, Boersma K, Linton SJ. Pain catastrophizing as repetitive negative thinking: a development of the conceptualization. Cogn Behav Ther. 2013;42(3):215-223.

58. Muthén LK, Muthén BO. Mplus user's guide (Eighth ed.). Los Angeles CA: Muthén \& Muthén. ; 2017. 
59. Schafer JL, Graham JW. Missing data: Our view of the state of the art. Psychol Methods. 2002;7(2):147-177.

60. Little TD. Longitudinal structural equation modeling. New York: The Guilford Press; 2013.

61. Hu LT, Bentler PM. Cutoff Criteria for Fit Indexes in Covariance Structure Analysis: Conventional Criteria Versus New Alternatives. Structural Equation Modeling-a Multidisciplinary Journal. 1999;6(1):1-55.

62. Wang J, Wang X. Structural Equation Modeling: Applications Using Mplus. Chichester: John Wiley \& Sons; 2012.

63. Bentler PM. Comparative Fit Indexes in Structural Models. Psychol Bull. 1990;107(2):238-246.

64. Wickrama KAS, Lee TK, O'Neal CW, Lorenz FO. Higher-order growth curves and mixture modeling with Mplus: A practical guide. New York, NY, US: Routledge/Taylor \& Francis Group; 2016.

65. Satorra A, Bentler PM. A scaled difference chi-square test statistic for moment structure analysis. Psychometrika. 2001;66(4):507-514.

66. Hamaker EL, Kuiper RM, Grasman RPPP. A critique of the cross-lagged panel model. Psychol Methods. 2015;20(1):102-116.

67. Preacher KJ, Rucker DD, Hayes AF. Addressing moderated mediation hypotheses: Theory, methods, and prescriptions. Multivariate Behavioral Research. 2007;42(1):185-227.

68. Linton SJ, Bergbom S. Understanding the link between depression and pain. Scandinavian Journal of Pain. 2011(2):47-54.

69. Arnow BA, Blasey CM, Constantino MJ, et al. Catastrophizing, depression and pain-related disability. Gen Hosp Psychiatry. 2011;33(2):150-156.

70. Hagger M. Avoiding the "déjà-variable" phenomenon: social psychology needs more guides to constructs. Front Psychol. 2014;5(52).

71. Campbell P, Bishop A, Dunn KM, Main CJ, Thomas E, Foster NE. Conceptual overlap of psychological constructs in low back pain. Pain. 2013;154(9):1783-1791.

72. Wolf EJ, Harrington KM, Clark SL, Miller MW. Sample Size Requirements for Structural Equation Models: An Evaluation of Power, Bias, and Solution Propriety. Educ Psychol Meas. 2013;76(6):913-934. 


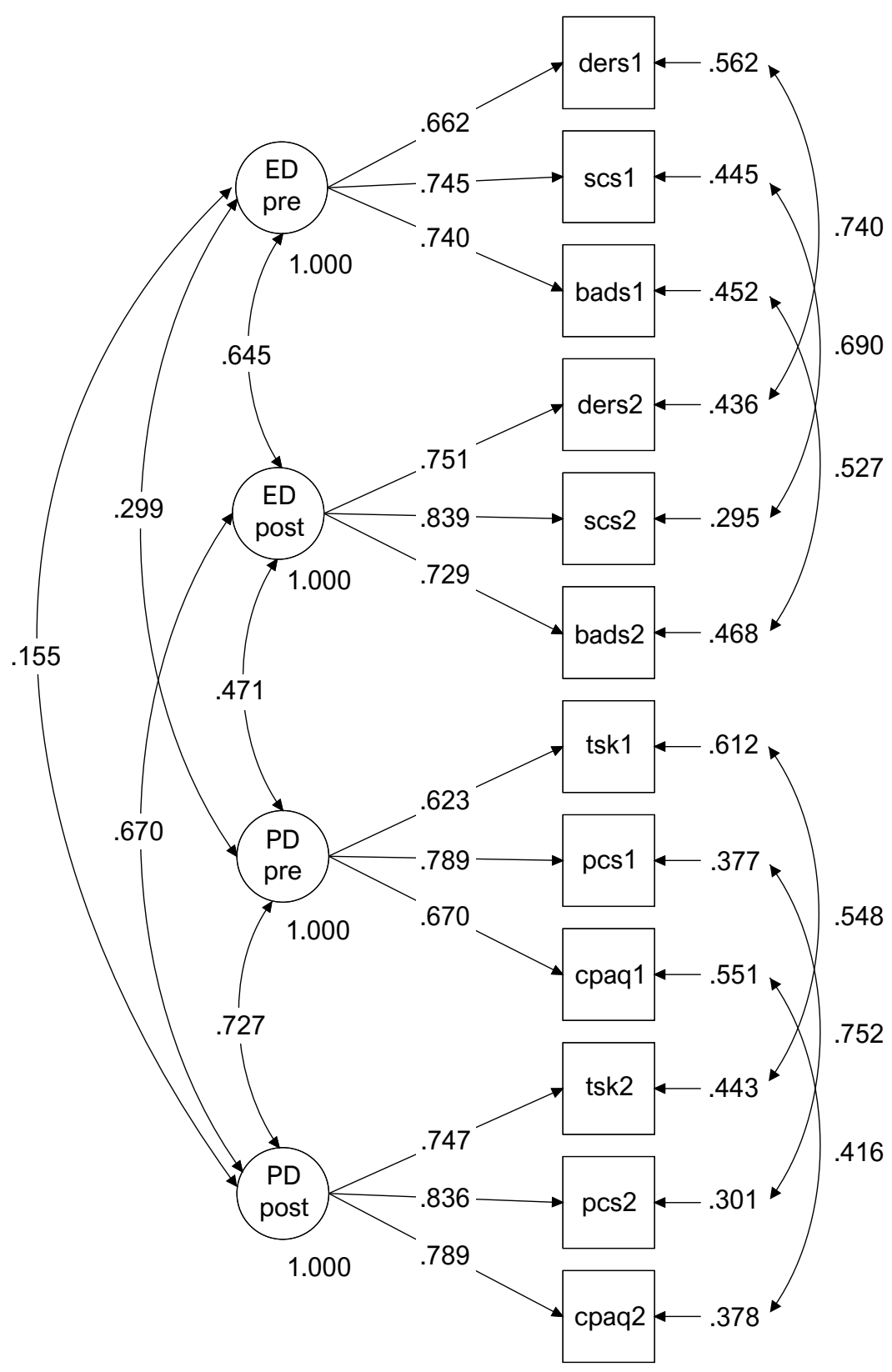

Supplementary figure. Longitudinal factor analysis model of the two latent mediators assessed at pretreatment (Time 1) and post-treatment (Time 2). Standardized robust maximum likelihood parameter estimates are shown. DERS; Difficulties in Emotion Regulation Scale, SCS; Self-Compassion Scale-12, BADS; Behavioral Activation for Depression Scale, TSK; Tampa Scale for Kinesiophobia-11, PCS; Paincatastrophizing scale, CPAQ; Chronic Pain Acceptance Questionnaire-8. Observed indicators SCS, BADS and CPAQ were reflected prior to analyses so that all factor loadings were positive. PDpre = pain-related dysregulation at pre-treatment; PDpost= pain-related dysregulation at post-treatment; EDpre = general emotion dysregulation at pre-treatment; EDpost = general emotion dysregulation at post-treatment. 